\title{
Conversión digital de texto entre sistemas Braille y Letra en tinta
}

\section{Digital text conversion between Braille and Letter in Ink systems}

\author{
CRUZ-LUNA, Manuel $\dagger^{*}$, HERNANDEZ-LUNA, Aldo, MARTINEZ-HERNANDEZ, Julio Cesar y \\ CRUZ-DOMINGUEZ, Adriana
}

Instituto Tecnológico Superior de Huauchinango

ID $1^{\text {er }}$ Autor: Manuel, Cruz-Luna / ORC ID: 0000-0002-0640-8926, Researcher ID Thomson: H-8709-2018, CVU CONACYT ID: 368159

ID $1^{\text {er }}$ Coautor: Aldo, Hernandez-Luna / ORC ID: 0000-0002-7717-5314, Researcher ID Thomson: I-1165-2018, CVU CONACYT ID: 441305

ID $2^{\text {do }}$ Coautor: Julio Cesar, Martinez-Hernandez / ORC ID: 0000-0001-9528-156X, Researcher ID Thomson: I-43342018, CVU CONACYT ID: 904537

ID $3^{\text {er }}$ Coautor: Adriana, Cruz-Dominguez / ORC ID: 0000-0002-1486-9343, Researcher ID Thomson: F-7415-2019, CVU CONACYT ID: 971865

DOI: $10.35429 /$ JCA.2019.10.3.23.28

Recibido Abril 30, 2019; Aceptado Junio 30, 2019

\section{Resumen}

La escritura es una forma de comunicación disponible para preservar información, ideas y conocimiento, para ser transmitido entre diversos grupos sociales y a través del tiempo de una generación hacia sus sucesoras. Para utilizar el sistema de Letra en tinta, se requiere que la persona tenga la capacidad de ver para reconocer los trazos que forman las letras y puedan obtener conocimiento; para el caso de las personas invidentes requieren de utilizar otro sentido, el tacto, para llevar a cabo la lectura de información utilizando el sistema Braille. Hoy en día se tiene una gran cantidad de información en formato digital, manipulada por medio de sistemas informáticos, con la característica que se requiere de la vista para poder leer dicha información. El desarrollo de este sistema de conversión permitirá integrar a las personas invidentes al mundo de la información digital, de forma que puedan acceder a textos de archivos, representarlos en sistema Braille y tengan la capacidad de leerlos sin ningún problema; además les permitirá plasmar sus propias emociones y conocimientos escribiéndolos en el sistema que ya conocen y sea convertido a sistema de Letra en tinta, para que posteriormente sea colocado en Internet y utilizado a nivel mundial

Texto digital, Braille, Conversión

\begin{abstract}
Writing is a communication way available to preserve information, ideas and knowledge, to be transmitted between different social groups and through the step of time of a generation towards its successors. To use the system of Letter in ink, it is required that the person can use the sight to recognize the lines and strokes that form the letters and can obtain knowledge; for blind people, they need to use another sense, in this case touch, to carry out the reading of information through the Braille system. Nowadays, there is a large amount of information in digital format, managed by informatics systems, where sight in needed to read that information. The development of this conversion system will allow blind people to be integrated into the world of digital information, so that they can access text files, represent them in Braille system and have the ability to read them without any problem; in addition, it will allow these people to express their own ideas, emotions and knowledge by writing them in the system they already know end be converted to the Letter in Ink system so that later, it can be placed on the Internet and used by anyone worldwide
\end{abstract}

Digital text, Braille, Conversion

Citación: CRUZ-LUNA, Manuel, HERNANDEZ-LUNA, Aldo, MARTINEZ-HERNANDEZ, Julio Cesar y CRUZDOMINGUEZ, Adriana. Conversión digital de texto entre sistemas Braille y Letra en tinta. Revista de Cómputo Aplicado. 2019, 3-10: 23-28

\footnotetext{
* Correspondencia al Autor (Correo electrónico: mcruzl@ hotmail.com)

$\dagger$ Investigador contribuyendo como primer Autor.
} 


\section{Introducción}

El ser humano requiere de la comunicación con sus semejantes para llevar a cabo una gran cantidad de actividades, desde una plática de convivencia hasta la transferencia de conocimientos entre generaciones. El proceso de comunicaciones se puede dar de dos formas diferentes, una cuando el emisor y el receptor se encuentran en espacios contiguos en el mismo tiempo, teniendo comunicaciones interactivas, donde el intercambio de información fluye en ambos sentidos de manera simultánea.

La otra forma de comunicación se da cuando el emisor y el receptor se encuentran en lugares o tiempos diferentes, lo que no permite tener una interacción tan rápida como la anterior. Para este último proceso se tuvieron que inventar algunas formas de preservar la información para que posteriormente sea recuperada por quienes estén interesados en adquirirla. La forma más común es la escritura, debido al tiempo que permanece la información sin que sufra alteraciones; la única característica que debe tener la persona que va a recuperar la información, es que debe tener desarrollado el sentido de la vista para poder leer la información que se encuentra impresa o representada en formatos digitales a través de pantallas o algún otro componente. A este sistema se le conoce como "Letra en tinta".

Para el caso de las personas invidentes, se requiere de un sistema de escritura en el que no se necesite de la vista para poder tener acceso a la información; este sistema, que se encuentra difundido a nivel mundial se conoce como Braille, debido a su inventor Louis Braille. Está formado por seis puntos para representar cada uno de los caracteres que se requieren en el idioma. Estos seis puntos se marcan sobre las hojas de papel, generando unas protuberancias en la ubicación de cada punto.

La forma de lectura es por medio del tacto, donde las personas pasan las yemas de los dedos sobre las protuberancias en las hojas, identifican la posición de cada una de ellas y van generando las letras, palabras, números y signos correspondientes al texto que se encuentra impreso. El objetivo de la presente investigación es el desarrollo de un algoritmo para realizar la traducción de texto entre los dos sistemas de escritura.

\section{Sistemas de Escritura}

\section{Letra en tinta}

Sistema utilizado para escritura, donde intervienen rasgos caligráficos para formar cada una de las letras, números y símbolos que contiene un documento escrito. Dependiendo de los rasgos que se tengan y la ubicación de cada uno de ellos, pueden dar un significado u otro a la información que se encuentra plasmada.

Para este sistema de escritura se tienen los siguientes símbolos disponibles en el lenguaje español:

\section{- $\quad 33$ letras mayúsculas \\ - $\quad 33$ letras minúsculas \\ - 10 números \\ - 13 signos aritméticos \\ - $\quad 10$ signos de puntuación}

La combinación de todos estos elementos da como resultado escritos con palabras, frases y oraciones que contienen ideas, pensamientos, logros, descubrimientos, historias o simplemente un texto que se quiera preservar.

\section{Braille}

Sistema utilizado para lectura y escritura donde se requiere únicamente del sentido del tacto para su aplicación. Se utiliza una serie de puntos ubicados en una cuadrícula de tres filas y dos columnas, dando como resultado seis posiciones diferentes; en cada una de estas posiciones puede o no haber un punto (una protuberancia sobre el papel), lo que nos da dos estados en cada una y un total de 64 combinaciones diferentes. Como ejemplo tenemos las combinaciones mostradas en la figura 1, donde los círculos negros representan las protuberancias en el papel y los blancos la hoja sin marcas.

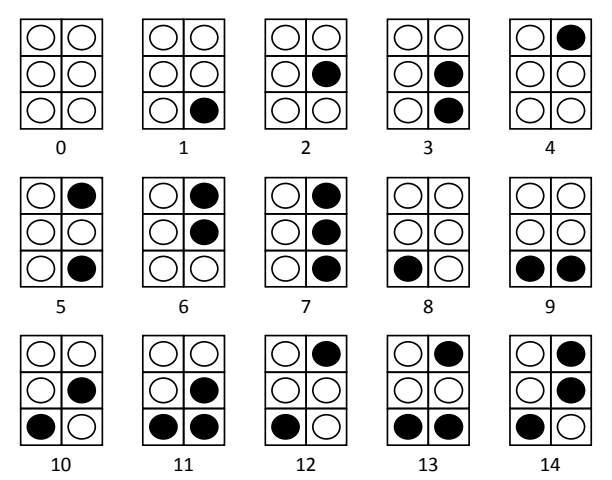

Figura 1 Caracteres en sistema Braille Fuente: Elaboración Propia
CRUZ-LUNA, Manuel, HERNANDEZ-LUNA, Aldo, MARTINEZHERNANDEZ, Julio Cesar y CRUZ-DOMINGUEZ, Adriana. Conversión digital de texto entre sistemas Braille y Letra en tinta. Revista de Cómputo Aplicado. 2019 


\section{Conversión entre Sistemas}

Debido a la diferencia en cuanto a la cantidad de símbolos entre los dos sistemas, se cuenta con un sistema de traducción entre ellos, donde la principal característica es que una combinación de puntos en el sistema Braille, representa varios símbolos en el sistema de Letra en tinta. La tabla uno muestra las combinaciones de puntos utilizadas para las primera letras del alfabeto, la primera columna muestra las combinaciones de puntos, si esa combinación se encuentra sin una combinación complementaria a su izquierda, representa las letras en minúscula, como se muestra en la columna dos. Si antes de cada combinación se coloca la complementaria que se encuentra ubicada en la parte superior de la tercera columna, entonces se está representando un digito numérico. Si antes de cada combinación se coloca la complementaria que se encuentra ubicada en la parte superior de la cuarta columna, entonces se está representando una letra en mayúscula. Si al inicio de cada palabra se coloca el indicador de mayúsculas una sola vez, se indica que la primera letra es mayúscula y las demás son minúsculas. Si al inicio de la palabra se coloca dos veces el indicador de mayúsculas, se indica que la palabra completa es en mayúsculas.

La tabla dos muestra un ejemplo del uso de los caracteres complementarios. Como se puede ver en la segunda columna, los últimos cuatro caracteres (combinaciones de puntos) en el sistema Braille son los mismos, lo único que cambia es la colocación de algún carácter antes de la palabra si es que se quiere colocar la inicial en mayúsculas, todo en mayúsculas o una serie de números.

\begin{tabular}{|c|c|c|c|}
\hline & & $\begin{array}{ll}0 \\
0\end{array}$ & $\begin{array}{ll}0 \\
00 \\
00\end{array}$ \\
\hline \begin{tabular}{|ll}
00 \\
00 \\
00
\end{tabular} & $\mathrm{a}$ & 1 & A \\
\hline$\frac{10}{\circ 9}$ & b & 2 & B \\
\hline \begin{tabular}{|l|}
00 \\
00 \\
00
\end{tabular} & $\mathrm{c}$ & 3 & $\mathrm{C}$ \\
\hline $\begin{array}{ll}00 \\
00\end{array}$ & $\mathrm{~d}$ & 4 & $\mathrm{D}$ \\
\hline \begin{tabular}{|l|}
00 \\
00 \\
00
\end{tabular} & $\mathrm{e}$ & 5 & $\mathrm{E}$ \\
\hline 00 & $\mathrm{f}$ & 6 & $\mathrm{~F}$ \\
\hline 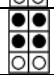 & $\mathrm{g}$ & 7 & $\mathrm{G}$ \\
\hline $\begin{array}{ll}00 \\
00 \\
00\end{array}$ & $\mathrm{~h}$ & 8 & $\mathrm{H}$ \\
\hline $\begin{array}{ll}0 \\
00\end{array}$ & $\mathrm{i}$ & 9 & I \\
\hline \begin{tabular}{|l|l}
0 \\
00 \\
00
\end{tabular} & $\mathrm{j}$ & 0 & $\mathrm{~J}$ \\
\hline
\end{tabular}

Tabla 1 Combinaciones de puntos para letras y números

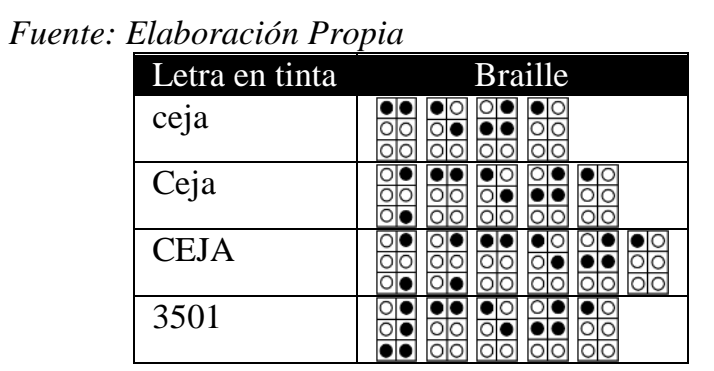

Tabla 2 Ejemplo de uso de caracteres complementarios Fuente: Elaboración Propia

\section{Metodología Aplicada}

Para el desarrollo del presente trabajo, se hizo uso de la metodología de desarrollo de software por prototipo, debido a que se pueden hacer las pruebas y modificaciones necesarias en un prototipo que se construye con todas las características que requiere el producto final. Las fases que se llevaron a cabo son:

1. Recolección y refinamiento de requisitos. Se hizo un listado de los requisitos que debe cumplir el prototipo, llevando a cabo un análisis y clasificación de los requisitos.

2. Diseño rápido. Se realizó el diseño de la solución para llevar a cabo la conversión de texto entre los dos sistemas, utilizando las ocho posiciones que tiene un caracter dentro de la computadora y las seis que tiene un caracter en el sistema Braille. En este paso fue necesario tomar en consideración las características particulares que tiene el sistema Braille para el uso de símbolos complementarios y aumentar con esto la cantidad de letras números y símbolos utilizados.

3. Construcción del prototipo. Se desarrolló el algoritmo para llevar a cabo la traducción de Letra en tinta hacia Braille, dando como resultado la posibilidad de hacerlo en ambos sentidos entre los dos sistemas de escritura.

4. Evaluación del prototipo por el cliente. Se llevó a cabo una verificación de los resultados obtenidos por el algoritmo a través de pruebas de escritorio, donde se colocaron una serie de textos con letras mayúsculas, minúsculas, números y símbolos como datos de entrada y se verificó el texto obtenido en sistema Braille. 
5. Refinamiento del prototipo. Un vez que el prototipo obtuvo la funcionalidad requerida, se trabajó en la elaboración de un programa para la automatización de las conversiones entre los sistemas de escritura.

6. Producto de ingeniería. Finalmente se obtuvo el resultado esperado, un sistema de conversión de texto de sistema Letra en tinta a sistema Braille.

\section{Algoritmo Utilizado}

Para la conversión de sistema Braille a Letra en tinta se requiere de la identificación de cada uno de los puntos que forman cada uno de los caracteres, ya sea por los que se encuentran marcados en la hoja por medio de un sistema de lectura con sensores, o bien, a través de un sistema de botones donde la persona vaya escribiendo en tiempo real cada uno de los caracteres que necesite para su documento.

Debido a que se tienen únicamente 6 puntos por caracter y dos estados, nos da un total de $2^{6}$, es decir, 64 combinaciones diferentes. Como se muestra en la figura 2 , a cada uno de los puntos se le asigna una posición dentro de los dígitos binarios de un byte y se almacena dentro de la secuencia de texto, posteriormente se verifica si es un caracter complementario o de un símbolo, si es un carácter significativo se almacena en el texto convertido, de lo contrario se espera al siguiente caracter para ser mostrado tomando como referencia la combinación de ambos símbolos. Tomando el valor final del caracter a utilizar, se hace la búsqueda dentro de la tabla de equivalencias (Tabla 3) para identificar el símbolo que se va a almacenar dentro del texto traducido.

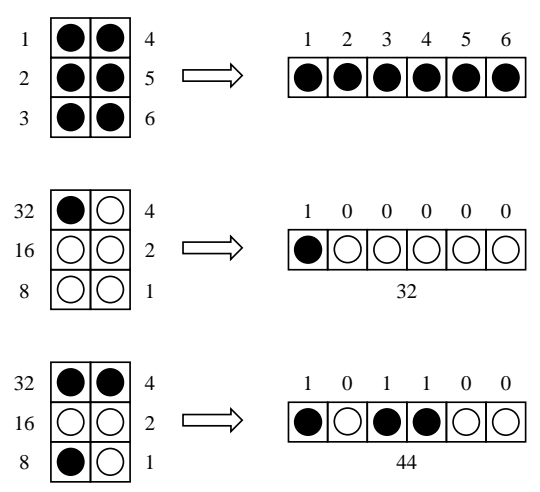

Figura 2 Conversión de Braille a bytes Fuente: Elaboración Propia
Se deben de almacenar las dos secuencias de caracteres identificados, por una parte, los que se van leyendo del documento en Braille y por otra parte los que se van generando como resultado de la traducción en sistema Letra en tinta.

\begin{tabular}{|c|c|c|c|c|c|}
\hline Letra & Valor & Letra & Valor & Letra & Valor \\
\hline$a$ & 32 & 1 & 56 & $\mathrm{~V}$ & 57 \\
\hline $\mathrm{b}$ & 48 & $\mathrm{~m}$ & 44 & $\mathrm{~W}$ & 23 \\
\hline $\mathrm{c}$ & 36 & $\mathrm{n}$ & 46 & X & 45 \\
\hline $\mathrm{d}$ & 38 & $\tilde{\mathrm{n}}$ & 55 & $y$ & 47 \\
\hline $\mathrm{e}$ & 34 & 0 & 42 & $\mathrm{Z}$ & 43 \\
\hline $\mathrm{f}$ & 52 & $\mathrm{p}$ & 60 & á & 59 \\
\hline$g$ & 54 & $q$ & 62 & é & 29 \\
\hline $\mathrm{h}$ & 50 & $\mathrm{r}$ & 58 & 1 & 12 \\
\hline $\mathrm{i}$ & 20 & $\mathrm{~s}$ & 28 & ó & 13 \\
\hline $\mathrm{j}$ & 22 & $\mathrm{t}$ & 30 & ú & 31 \\
\hline $\mathrm{k}$ & 40 & $\mathrm{u}$ & 41 & $\ddot{\mathrm{u}}$ & 51 \\
\hline
\end{tabular}

Tabla 3 Equivalencias entre letras y valores de bytes en Braille

Fuente: Elaboración Propia

Para realizar al conversión de Letra en tinta hacia Braille, se va a tomar cada uno de los caracteres que se encuentran en el documento, si se trata de una letra minúscula, se busca su equivalencia en bytes directamente en la tabla de Equivalencias y se almacena en el texto convertido; si se trata de una letra mayúscula, se lee el siguiente caracter del texto, si es también una mayúscula, significa que toda la palabra es en mayúsculas y se coloca el caracter complementario dos veces, continuando con cada una de las letras de la palabra, convirtiéndolas en minúsculas y buscando su equivalencia en bytes dentro de la tabla correspondiente; si el segundo caracter se encuentra en minúscula, significa que nada más la inicial de la palabra es en mayúscula y se coloca un solo caracter complementario de mayúsculas, se convierte la primera letra en minúscula y se busca su equivalencia en bytes dentro de la tabla correspondiente, las demás letras de la palabra se buscarán de manera directa en la tabla de equivalencias.

En caso de que se trate de números, se coloca inicialmente el caracter complementario para números y cada uno de los dígitos se busca dentro de la tabla de equivalencias para obtener el valor en byte que le corresponde.

En caso de que sean caracteres adicionales, se busca su valor en bytes directamente en la tabla de equivalencias. 
La cadena de bytes que se obtenga como resultado de la conversión de Letra en tinta a Braille, se utilizará para mostrar la información en algún sistema que tenga la capacidad de manipular elementos físicos, donde las personas invidentes puedan percibir por medio del tacto las protuberancias que requieren para identificar textos, puedan leerlo y logren su comprensión sin ningún problema.

\section{Resultados Obtenidos}

Para la realización de pruebas del algoritmo de traducción, se genera una aplicación local para escritorio, donde se coloca como dato de entrada una frase utilizando el sistema Letra en tinta y se obtiene como resultado la secuencia de números que representa los bytes de cada letra en sistema Braille como se aprecia en la tabla 4. Se realizó un proceso adicional para obtener la combinación de puntos para byte, dando como resultado los caracteres correctos en Braille.

\begin{tabular}{|c|c|}
\hline Letra en tinta & Valores numéricos \\
\hline $\begin{array}{l}\text { Este texto es la } \\
\text { prueba número } \\
12 \text { para } \\
\text { conversiones de } \\
\text { Letra en tinta a } \\
\text { Braille }\end{array}$ & $\begin{array}{l}5,34,28,30,34,030,34,45,30, \\
42,0,34,28,0,56,32,0,60,58, \\
41,34,48,32,0,46,31,44,34,58, \\
42,0,15,32,33,0,60,32,58,32, \\
036,42,46,57,34,58,28,20,42, \\
46,34,28,0,38,34,0,5,56,34, \\
30,58,32,0,34,46,0,30,20,46, \\
30,32,0,32,0,5,48,48,32,20, \\
56,56,34\end{array}$ \\
\hline
\end{tabular}

Tabla 4 Conversión de Letra en tinta a Braille Fuente: Elaboración Propia

En el otro sentido, se tomó un tex to de un documento en Braille, se calculó el valor en bytes de cada letra y se agregó como entrada al algoritmo propuesto, dando como resultado el texto que se muestra en la tabla 5.

Valores numéricos

$5,34,56,0,28,20,28,30,34$ $44,32,0,5,48,58,32,20,56$, $56,34,0,34,28,0,41,46,0$, $56,34,46,54,41,32,22,34,0$, 41, 30, 20, 56, 20, 43, 32, 38, $42,0,60,42,58,0,44,34,38$, $20,42,0,38,34,56,0,30,32$, $36,30,42$

Tabla 5 Conversión de Braille a Letra en tinta Fuente: Elaboración Propia

\section{Conclusiones}

Este algoritmo propuesto para la conversión entre textos de los sistemas Letra en tinta y Braille es de gran ayuda para las personas invidentes, debido a que los acerca a los documentos y la información que puede estar disponible en cualquier documento de texto. Además de que les permite expresar sus ideas, sentimientos, emociones y conocimientos sobre algún tema en particular y les da la posibilidad de convertir sus textos a un sistema que pueda ser utilizado por la mayoría de personas en cualquier dispositivo electrónico con la capacidad de manipulación de archivos de texto; logrando con esto que todas las personas tengan acceso a la información que se encuentra almacenada de manera digital, sin importar si pueden hacer uso o no de su sentido de la vista.

\section{Sugerencias}

Para la aplicación de este algoritmo de conversión de texto, se requiere de un sistema informático y mecatrónico que permita a una persona invidente llevar a cabo la escritura, lectura e impresión de texto en sistema Braille y, su correspondiente conversión a sistema Letra en tinta; que tenga además la capacidad de guardar el texto en dispositivos de almacenamiento extraíble y pueda ser abierto en cualquier computadora como un archivo de texto, se modifique, guarde y lo pueda volver a abrir la persona invidente para que lea los cambios y pueda contribuir con otras personas a la escritura de documentos sin ningún problema.

\section{Referencias}

(España Patente $n^{\circ}$ ES2660640B1, 2019)

Barrios, T. H., Marín, M. B. y Torrente, N. (2017). XIX Workshop de Investigadores en Ciencias de la Computación: El Uso de la Tecnología para la Inclusión de los Disminuidos Visuales en las Aulas.

Gonzalez-García, L. (1999). III Jornadas Científicas de Investigación sobre Personas con Discapacidad: Comprensión lectora en lectura braille. 
Hernández, C., Pedraza, L. F. y López, D. (2011). Revista de salud pública: Dispositivo tecnológico para la optimización del tiempo de aprendizaje del lenguaje Braille en personas invidentes.

Lorenzo Cadarso, P. L. y Sánchez Sánchez, P. Puertas a la lectura: Leer sin ver.

Martínez-Liebana, I. y Polo Chacón, D. (2004). Guía didáctica para lectoescritura Braille.

Pantaleo, G. y Rinaudo L. (2015). Ingeniería de software. Alfaomega.

Pauta, J. A. A., Vélez, E. P. and Serpa-Andrade, L. (2016). 2016 IEEE International Autumn Meeting on Power, Electronics and Computing: Braille teaching electronic prototype.

Perez-Castro, J. (2016). Sinectica: La inclusión de las personas con discapacidad en la educación superior en México.

Serrano Marrugan, I. y Palomares Ruiz, A. (2013). Eticanet: La accesibilidad en las TIC para alumnos con discapacidad visual. Un reto para el profesorado.

Serrano Marugán, I., Palomares Ruiz, A. y Garrote Rojas, D. (2013). ENSAYOS, Revista de la Facultad de Educación de Albacete: Propuestas innovadoras para favorecer el uso de las TIC y propiciar la inclusión educativa del alumnado con discapacidad visual".

Silva Sprock, A., Ponce, J. C. (2014). Rbraile: REA basado en reglas inteligentes para lectura braille

Simón, C., Ochaíta, E. y Huertas, J. A. (1995). Comunicación, lenguaje y educación: El sistema Braille, bases para su enseñanza-aprendizaje.

Sommerville, I. (2011). Ingeniería de software. Pearson. 